\title{
Atividade antimicrobiana in vitro da cefpiroma em comparação com outros beta-lactâmicos de amplo espectro contra 804 amostras clínicas de nove hospitais brasileiros
}

\author{
H.S. SAder ${ }^{1}$, C.M.F. Mendes ${ }^{2}$, A. Montelli ${ }^{3}$, J . Sampalo ${ }^{4}$, A.J .A. Segura ${ }^{5}$, G.L.F. KesselRing ${ }^{6}$, L. Costa ${ }^{7}$, \\ J.E.F. RIBEIRO ${ }^{8}$, E.MAMIZUKA ${ }^{9}$, I. MIMIÇA ${ }^{10}$
}

${ }^{1}$ UniversidadeF ederal deSão Paulo-E scola Paulista de Medicina, SãoPaulo, SP; ${ }^{2}$ F aculdade de Medicina da Universidade deSão Paulo, São Paulo, SP; ${ }^{3}$ Universidade E stadual Paulista - UNESP, Botucatu, SP; ${ }^{4}$ Laboratório Lâmina, Rio de J aneiro, RJ ; ${ }^{5} \mathrm{H}$ ospital de Base de Brasília, Brasília, DF; ${ }^{6}$ Médico-Consultor Científico, São Paulo, SP; 7 Universidade Federal do Paraná, Curitiba, PR; ${ }^{8}$ Santa Casa de Misericórdia de Belo Horizonte, Belo Horizonte, MG; ${ }^{9}$ F aculdade de Ciências F armacêuticas da U niversidade de São Paulo, São Paulo, SP; ${ }^{10}$ Santa Casa de Misericórdia de São Paulo, São Paulo, SP.

RESUMO - Овј етIVo. Avaliar a atividade in vitroda cefalosporina de quarta geração, cefpiroma em comparação com ceftazi dima, ceftriaxona, cefotaxima e imipenem em um estudo multicêntrico envolvendo nove hospitais de seis cidades em quatro estados.

Material e Métodos. Foram estudadas 804 amostras clínicas isoladas em pacientes internados em unidades de terapia intensiva ou unidades de oncohematologia. As amostras foram coletadas no período de junho a novembro de 1995, isto é, antes da cefpiroma estar disponível comercialmente no Brasil, e testadas através do método de microdiluição em placas conforme descrito pelo National Committee for Clinical Laboratory Standards (NCCLS). Todas as amostras resistentes à cefpiroma foram retestadas utilizando-se o E-test.

Resultados. Contra as amostras de enterobactérias $(n=344)$, a cefpiroma apresentou atividade de 2 a 32 vezes superior àquela apresentada pelas cefalosporinas de terceira geração (CTGs) e semeIhante aquela apresentada pelo imipenem. As porcentagens de enterobactérias sensíveis foram: $88 \%$ para a cefpiroma, $69 \%$ para as CTGs e 96\% para o imipenem. O espectro de ação da cefpiroma foi maior ou igual ao do imipenem contra as espécies Citrobacter freundii, E. aerogenes, Morganella morganii e Serratia marces-

\section{INTRODUÇÃO}

Uma vez que a produção de $\beta$-lactamases é o mecanismo mais importante e prevalente de resistência aos antibióticos $\beta$-lactâmicos em bactérias gram-negativas, o desenvolvimento de novas drogas tem sido direcionado para o desenho de compostos mais estáveis à hidról ise por essas enzimas. As $\beta$-lactamases cromossômi cas da Classe I (Rich- cens. Contra Acinetobacter sp. ( $n=77)$, a cefpiroma foi ligeiramente mais ativa que a ceftazidima, porém as porcentagens de resistência foram muito altas para esses compostos (84\% e $88 \%$ respectivamente). As atividades da cefpiroma, ceftazidima e imipenem foram semelhantes contra Pseudomonas aeruginosa $(n=128)$, com MIC50/ porcentagem de sensibilidade de $8 / 59 \%$, $8 / 62 \%$ e $4 /$ $62 \%$ respectivamente. Contra bactérias aeróbias gram-positivas, a cefpiroma foi de 4 a 16 vezes mais ativa que as CTGs. Contra S. epidermidis e outras espécies de estafilococos coagulase-negativos a cefpiroma foi ligeiramente superior ao imipenem, porém, contra as outras espécies de bactérias gram-positivas avaliadas, 0 imipenem apresentou atividade um pouco superior.

ConclusÃo: Os resultados desse estudo sugerem que, no Brasil, a cefpiroma apresenta espectro de ação superior ao das CTGs contra bactérias gramnegativas (Enterobacteriaceae e não-fermentadares) e gram-positivas e semelhante ao do imipenem contra algumas espécies de enterobactérias e contra $P$. aeruginosa.

UNITERMOS: Cefpiroma. Atividade in vitro. Cefalosporinas. Sensibilidade a antimicrobianos. Bactérias hospitalares. Unidades de terapia intensiva. mond - Sykes tipo I, Bush grupo 1) representam as $\beta$-lactamases mais importantes produzidas por bactérias gram-negativas ${ }^{1-2}$. Essas enzimas são provavel mente produzidas por todas as bactérias gram-negativas, porém o modo de produção varia nas diferentes espécies, afetando a expressão fenotípica ou clínica da resistência ${ }^{3}$. Escherichia coli e Klebsiella sp. conseguem produzir apenas quantidades pequenas de enzimas e a produção 
não parece ser afetada pelo uso ou presença de $\beta$-lactâmi cos, ou seja, não é induzível. Em outras espécies como Enterobacter sp., Citrobacter sp., Serratia sp., Providencia sp., Morganella sp. e $P$ seudomonas sp., essas $\beta$-lactamases também são normalmente produzidas em pequenas quantidades. Porém a produção pode aumentar significativamente (freqüentemente 100 a 1.000 vezes) quando os organismos são expostos a alguns $\beta$ lactâmicos. Além disso, essas espécies podem apresentar mutações (taxas variam dependendo da espécie) que levam a produção de grandes quantidades de $\beta$-lactamases da Classe I de maneira constitutiva, tornando a amostra resistente aos $\beta$ lactâmi cos mais estáveis, inclusive CTGs. A seleção de clones mutantes tem sido descrita após uso demasiado ou mesmo adequado de certas cefalosporinas tanto nível hospitalar, quanto comunitário $0^{3-5}$.

As $\beta$-lactamases de espectro ampliado (ESBL) representam outro grupo de $\beta$-lactamases clinicamente importantes produzidas por enterobactérias $^{4-5}$. As ESBLs são mediadas por genes plasmidiais, não induzíveis, e conferem resistência às CTGs, como cefotaxima e ceftazidima, e ao aztreonam. Essas enzimas são classificadas como grupo $2 b^{\prime}$ de Bush $^{1}$ e foram primeiramente descritas em Klebsiella sp., porém já foram demonstradas em $E$. coli e Proteus sp ${ }^{6}$. O grau de resistência que essas enzimas conferem pode variar consideravelmente de um $\beta$-lactâmico para outro e essa resistência é dificilmente detectada pelos métodos tradicionais de testes de sensibilidade aos antimicrobianos ${ }^{7}$.

A cefpiroma é uma nova cefal osporina, que devido ao seu amplo espectro de ação foi classificada como uma cefalosporina de quarta geração ${ }^{8}$. Sua estrutura molecular é semelhante àquela das CTGs, porém foram incluídas algumas modificações nas moléculas que propiciam uma maior estabilidade à hidrólise pelas $\beta$-lactamases, principalmente aquel as da Classe I, e maior permeabilidade pela membrana externa de bactérias gram-negativas $^{9,10}$. Estudos multicêntricos internacionais têm mostrado que esse composto apresenta maior atividade contra enterobactérias que as CTGs ${ }^{11-13}$. Sua atividade contra Pseudonomas sp. é semeIhante àquela demonstrada pela ceftazidima, podendo variar de um hospital para outro, enquanto que sua atividade contra cocos gram-positivos é semelhante àquela apresentada pelas cefalosporinas de primeira geração, que são as cefalosporinas mais ativas contra esse grupo de bactéri$a^{13,14}$. O objetivo do presente estudo foi avaliar o espectro de ação da cefpi roma em comparação com outros $\beta$-lactâmicos de amplo espectro, inclusive carbapenens. Apesar de estudos semelhantes já terem si do real izados em outros países, é importante a avaliação de amostras bacterianas isoladas em nosso país, uma vez que existe variação importante na prevalência de microrganismos produtores de diferentes $\beta$-lactamases nos diferentes locais.

\section{MATERIAIS E MÉTODOS}

F oram avaliadas 804 amostras clínicas isoladas somente em unidades de terapia intensiva (UTIs) e em unidades de oncohematologia de nove hospitais do Brasil, no período de junho a novembro de 1995. Essas uni dades foram sel ecionadas pel o fato de apresentar taxas al tas de infecções por bactérias multirresistentes, para as quais novas opções de tratamento podem ser necessárias. F oram avaliadas amostras dos seguintes hospitais: Hospital São Paulo (100 amostras), Hospital das Clínicas da Faculdade de Medicina da Universidade de São Paulo-USP (100 amostras) Santa Casa de Misericórdia de São Paulo (101 amostras), Hospital das Clínicas de Botucatu (100 amostras), Laboratório Lâmina (102 amostras de vários hospitais do Rio de J aneiro), Hospital de Base de Brasília (100 amostras), Universidade Federal do Paraná (72 amostras), Hospital Universitário da USP (40 amostras) e Santa Casa de Misericórdia de Belo Horizonte (89 amostras).

F oram avaliadas 570 amostras clínicas de bactérias gram-negativas, sendo 349 enterobactérias e 221 não-fermentadoras e 234 amostras clínicas de bactérias gram-positivas. A distribuição das amostras foi a seguinte: Citrobacter freundii (11 amostras), Enterobacter aerogenes (27 amostras), E. cloacae (44 amostras), Enterobacter sp. (24 amostras), E. coli (79 amostras), K. pneumoniae (96 amostras), Klebsiella sp. (15 amostras), Morganella morganii (10 amostras), Proteus sp. (18 amostras), Serratia marcescens (20 amostras), outras enterobactérias (5 amostras), Pseudomonas aeruginosa (128 amostras), Acinetobacter sp. (77 amostras), outras bactérias não-fermentadoras (16 amostras), S. aureus (141 amostras), S. epidermidis (11 amostras), estafi lococos coagulase negativo (17 amostras), Streptococcus sp. (15 amostras), Enterococcus faecalis (40 amostras), e outras bactérias gram-positivas (10 amostras).

As amostras foram testadas através do método de microdiluição em placa utilizando o Sistema Sensitrite (Sensititre Ltda, Inglaterra) e a metodologia preconizada pelo National Committee for Clinical Laboratory Standards $(\mathrm{NCCLS})^{15}$. Foi avaliada a concentração inibitória mínima (CIM) para os seguintes antimicrobianos: cefpiroma e 
Tabela 1 - Atividade in vitro dos $5 \beta$ - lactâmicos avaliados contra enterobactérias

\begin{tabular}{|c|c|c|c|c|}
\hline $\begin{array}{l}\text { Organismo } \\
\text { (n) }\end{array}$ & Antimicrobiano & $\begin{array}{l}\text { MICs } \\
\text { MIC50 }\end{array}$ & $\begin{array}{l}\mathrm{ug} / \mathrm{ml} \text { ) } \\
\text { MIC90 }\end{array}$ & $\begin{array}{l}\text { Porcentagem } \\
\text { suscetívela(\%) }\end{array}$ \\
\hline $\begin{array}{l}\text { Citrobacter } \\
\text { freundii } \\
(11)\end{array}$ & $\begin{array}{l}\text { Cefpiroma } \\
\text { Caftazidima } \\
\text { Ceftriaxona } \\
\text { Cefotaxima } \\
\text { Imipenem }\end{array}$ & $\begin{array}{r}0,25 \\
1 \\
1 \\
1 \\
0,25\end{array}$ & $\begin{array}{r}1 \\
16 \\
8 \\
8 \\
1\end{array}$ & $\begin{array}{r}100 \\
82 \\
91 \\
91 \\
100\end{array}$ \\
\hline $\begin{array}{l}\text { Enterobacter } \\
\text { aerogenes } \\
(27)\end{array}$ & $\begin{array}{l}\text { Cefpiroma } \\
\text { Caftazidima } \\
\text { Ceftriaxona } \\
\text { Cefotaxima } \\
\text { Imipenem }\end{array}$ & $\begin{array}{r}\leq 0,06 \\
0,5 \\
0,25 \\
0,25 \\
0,5\end{array}$ & $\begin{array}{r}8 \\
64 \\
64 \\
>64 \\
2\end{array}$ & $\begin{array}{l}93 \\
74 \\
67 \\
67 \\
92\end{array}$ \\
\hline $\begin{array}{l}\text { Enterobacter } \\
\text { cloacae } \\
(44)\end{array}$ & $\begin{array}{l}\text { Cefpiroma } \\
\text { Ceftazidima } \\
\text { Ceftriaxona } \\
\text { Cefotaxima } \\
\text { Imipenem }\end{array}$ & $\begin{array}{r}0,5 \\
8 \\
8 \\
16 \\
0,5\end{array}$ & $\begin{array}{r}8 \\
>64 \\
>64 \\
>64 \\
2\end{array}$ & $\begin{array}{l}90 \\
50 \\
52 \\
50 \\
98\end{array}$ \\
\hline $\begin{array}{l}\text { Enterobacter } \\
\text { sp. (24) }\end{array}$ & $\begin{array}{l}\text { Cefpiroma } \\
\text { Ceftazidima } \\
\text { Ceftriaxona } \\
\text { Cefotaxima } \\
\text { Imipenem }\end{array}$ & $\begin{array}{r}2 \\
16 \\
32 \\
32 \\
1\end{array}$ & $\begin{array}{r}>64 \\
>64 \\
>64 \\
>64 \\
4\end{array}$ & $\begin{array}{l}71 \\
46 \\
33 \\
33 \\
92\end{array}$ \\
\hline $\begin{array}{l}\text { Escherichia } \\
\text { coli (79) }\end{array}$ & $\begin{array}{l}\text { Cefpiroma } \\
\text { Ceftazidima } \\
\text { Ceftriaxona } \\
\text { Cefotaxima } \\
\text { Imipenem }\end{array}$ & $\begin{array}{r}\leq 0,06 \\
0,25 \\
\leq 0,06 \\
0,12 \\
0,12\end{array}$ & $\begin{array}{r}2 \\
8 \\
8 \\
16 \\
1\end{array}$ & $\begin{array}{r}92 \\
60 \\
90 \\
86 \\
100\end{array}$ \\
\hline $\begin{array}{l}\text { Klebsiella } \\
\text { pneumoniae } \\
(96)\end{array}$ & $\begin{array}{l}\text { Cefpiroma } \\
\text { Caftazidima } \\
\text { Ceftriaxona } \\
\text { Cefotaxima } \\
\text { Imipenem }\end{array}$ & $\begin{array}{r}2 \\
8 \\
8 \\
8 \\
0,25\end{array}$ & $\begin{array}{r}32 \\
>64 \\
>64 \\
>64 \\
2\end{array}$ & $\begin{array}{l}78 \\
53 \\
57 \\
58 \\
93\end{array}$ \\
\hline $\begin{array}{l}\text { Klebsiella sp. } \\
(15)^{\mathrm{b}}\end{array}$ & $\begin{array}{l}\text { Cefpiroma } \\
\text { Caftazidima } \\
\text { Ceftriaxona } \\
\text { Cefotaxima } \\
\text { Imipenem }\end{array}$ & $\begin{array}{r}1 \\
8 \\
4 \\
1 \\
0,25\end{array}$ & $\begin{array}{r}>64 \\
64 \\
>64 \\
64 \\
2\end{array}$ & $\begin{array}{l}87 \\
67 \\
67 \\
80 \\
93\end{array}$ \\
\hline $\begin{array}{l}\text { Morganella } \\
\text { morganii }(10)\end{array}$ & $\begin{array}{l}\text { Cefpiroma } \\
\text { Ceftazidima } \\
\text { Ceftriaxona } \\
\text { Cefotaxima } \\
\text { Imipenem }\end{array}$ & $\begin{array}{r}\leq 0,06 \\
0,12 \\
\leq 0,06 \\
0,12 \\
1\end{array}$ & $\begin{array}{l}8 \\
2 \\
4 \\
2 \\
4\end{array}$ & $\begin{array}{r}90 \\
90 \\
100 \\
90 \\
90\end{array}$ \\
\hline $\begin{array}{l}\text { Proteus } \\
\text { sp. (18) }\end{array}$ & $\begin{array}{l}\text { Cefpiroma } \\
\text { Ceftazidima } \\
\text { Ceftriaxona } \\
\text { Cefotaxima } \\
\text { Imipenem }\end{array}$ & $\begin{array}{r}0,12 \\
0,12 \\
\leq 0,06 \\
\leq 0,06 \\
1\end{array}$ & $\begin{array}{r}1 \\
0,25 \\
0,25 \\
0,25 \\
4\end{array}$ & $\begin{array}{r}90 \\
94 \\
94 \\
94 \\
100\end{array}$ \\
\hline $\begin{array}{l}\text { Serratia } \\
\text { marcescens } \\
(20)^{\mathrm{d}}\end{array}$ & $\begin{array}{l}\text { Cefpiroma } \\
\text { Ceftazidim } \\
\text { Ceftriaxona } \\
\text { Cefotaxima } \\
\text { Imipenem }\end{array}$ & $\begin{array}{r}0,12 \\
0,5 \\
0,25 \\
0,5 \\
0,5\end{array}$ & $\begin{array}{r}1 \\
16 \\
16 \\
32 \\
4\end{array}$ & $\begin{array}{r}100 \\
85 \\
85 \\
80 \\
90\end{array}$ \\
\hline $\begin{array}{l}\text { Outras } \\
\text { enterobactérias } \\
(5)^{d}\end{array}$ & $\begin{array}{l}\text { Cefpiroma } \\
\text { Ceftazidima } \\
\text { Ceftriaxona } \\
\text { Cefotaxima } \\
\text { Imipenem }\end{array}$ & $\begin{array}{r}0,25 \\
1 \\
0,12 \\
0,25 \\
0,25\end{array}$ & $\begin{array}{l}1 \\
1 \\
1 \\
1 \\
1\end{array}$ & $\begin{array}{l}100 \\
100 \\
100 \\
100 \\
100\end{array}$ \\
\hline \multicolumn{5}{|c|}{$\begin{array}{l}\text { a Suscetibidade definida como MIC ug/ml: } \leq 8 \text { para as cefalosporinas e } \leq 4 \\
\text { para o Imipenem. } \\
\text { b Incluindo Klesbsiella oxytoca ( } 11 \text { amostras), Klebsilla sp. (4 amostras). } \\
\text { c Incluindo Proteus mirabilis ( } 11 \text { amostras) e } P \text {. vulgaris ( } 7 \text { amostras). } \\
\text { d Incluindo Kluyera sp. ( } 1 \text { amostra), Providencia alcalifaciens ( } 1 \text { amostra), } \\
\text { Salmonella enteritidis (2 amostras), Serratia liquefaciens (1 amostra). }\end{array}$} \\
\hline
\end{tabular}

cefotaxima (Hoechst Marion Roussel), ceftazidima (Glaxo Wellcome Inc.), ceftriaxona (Roche), I mipenem (Merck Sharp \& Dowel).

\section{RESULTADOS E DISCUSSÃO}

Os resultados do estudo encontram-se resumidos nas tabelas 1 a 4 . A concentração de antimicrobiano capaz de inibir 50\% das amostras (MIC50) nos mostra a potência do antimicrobiano contra uma determinada espécie (ou grupo de espécies), pois não sofre a influência de cepas mutantes que tenham desenvolvido resistência, exceto, é claro, quando mais de $50 \%$ das amostras forem mutantes resistentes. A concentração capaz de inibir $90 \%$ das amostras (MIC90), por outro lado, nos mostra o grau de resistência desenvolvido pela espécie avaliada, enquanto que a porcentagem de amostras sensíveis nos mostra o espectro do antimicrobiano contra uma determinada espécie (ou grupo de espécies).

Contra enterobactérias ( $n=349$, tabela 1$)$ a cefpiroma apresentou atividade de 2 a 32 vezes superior àquela apresentada pelas CTGs e similar àquela apresentada pelo imipenem. A porcentagem de enterobactérias sensíveis à cefpiroma, CTGs (ceftazidima, cefotaxima e ceftriaxona apresentaram valores idênticos) e à imi penem foram, respectivamente: $88 \%, 69 \%$ e $96 \%$. A cefpiroma apresentou espectro de ação mai or ou igual àquele apresentado pelo imipenem contra as seguintes espécies: Citrobacter freundii, Enterobacter aerogenes, Morganella morganii e Serratia marcescens. Contra essas espécies, a cefpiroma apresentou potência e espectro bastante superi or es às cefal osporinas de terceira geração

Os resultados da avaliação desses $\beta$-lactâmicos para as bactérias gram-negativas não-fermentadoras mostraram resultados muito interessantes (tabela 2). Pseudomonas aeruginosa representa uma importante causa de infecções nas unidades escol hidas para se coletar amostras para o estudo (UTI e oncohematologia). Além de alta freqüência, essas infecções por $P$. aeruginosa apresentam alta taxa de mortalidade. Em muitos hospitais, as CTGs com atividade anti-pseudomonas (como ceftazidima por exemplo) são os antimicrobianos mais utilizados para tratamento empírico (antes do antibiograma) de infecções graves causadas por $P$. aeruginosa, sendo que o imi penem é reservado para amostras sensíveis somente a esse antimicrobiano. No presente estudo, esses dois $\beta$-lactâmicos (ceftazidima e imipenem) apresentaram espectro de ação idênticos para $P$. aeruginosa $(62 \%$ de amostras sensíveis), o qual foi muito semelhante 
Tabela 2 - Atividade in vitro dos 5 -lactâmicos avaliados contra bactérias não-fermentadores

\begin{tabular}{|c|c|c|c|c|}
\hline \multirow{5}{*}{$\begin{array}{l}\text { Organismo } \\
\qquad \begin{array}{l}(\mathbf{n}) \\
\text { Pseudomonas } \\
\text { aeruginosa } \\
(128)\end{array}\end{array}$} & \multirow{2}{*}{ Antimicrobiano } & \multicolumn{2}{|c|}{$\operatorname{MICs}(\mu \mathrm{g} / \mathrm{ml})$} & \multirow{2}{*}{$\begin{array}{l}\text { Porcentagem } \\
\text { suscetívela(\%) }\end{array}$} \\
\hline & & MIC50 & MIC90 & \\
\hline & Cefpiroma & 8 & 64 & 59 \\
\hline & Ceftazidima & 8 & 64 & 62 \\
\hline & Ceftriaxona & 64 & $>64$ & 13 \\
\hline & Cefotaxima & 64 & $>64$ & 9 \\
\hline & Imipenem & 4 & 16 & 62 \\
\hline Acinetobacter & Cefpiroma & 32 & $>64$ & 26 \\
\hline sp. $(77)^{\mathrm{b}}$ & Ceftazidima & 64 & $>64$ & 22 \\
\hline & Ceftriaxona & $>64$ & $>64$ & 18 \\
\hline & Cefotaxima & $>64$ & $>64$ & 20 \\
\hline & Imipenem & 1 & 16 & 86 \\
\hline Outras & Cefpiroma & 32 & $>64$ & 25 \\
\hline bactérias não & Ceftazidima & 8 & $>64$ & 56 \\
\hline fermentadoras & Ceftriaxona & $>64$ & $>64$ & 19 \\
\hline$(16)^{c}$ & Cefotaxima & $>64$ & $>64$ & 19 \\
\hline & Imipenem & 16 & $>64$ & 31 \\
\hline \multicolumn{5}{|c|}{$\begin{array}{l}\text { a Suscetibilidade definida como MIC } \mu \mathrm{g} / \mathrm{ml}: \leq 8 \text { para as cefalosporinas e } \\
<4 \text { para o Imipenem } \\
\text { b Incluindo } A \text {. baumannii ( } 54 \text { amostras), A. calcoaceticus (19 amostras) e } \\
\text { Acinetobacter sp. ( } 4 \text { amostras) } \\
\text { c Incluindo Pseudomonas stutzeri ( } 1 \text { amostra), P. fluorescens } \\
\text { ( } 1 \text { amostra), P. cepacia ( } 2 \text { amostras), P. alcaligenes ( } 1 \text { amostra), } \\
\text { P. putida ( } 1 \text { amostra), Xanthomonas maltophilia ( } 7 \text { amostras) e } \\
\text { Flavobacterium meningo-septicum ( } 2 \text { amostras) }\end{array}$} \\
\hline
\end{tabular}

àquele apresentado pela cefpiroma (59\%), mostrando que esses três $\beta$-lactâmicos possam apresentar papéis muito semel hantes na terapêutica empírica de infecções graves onde há suspeita de $P$. aeruginosa.

O outro achado interessante com relação às bactérias gram-negativas não-fermentadoras foi a baixa sensibilidade das amostras de Acinetobacter sp. A freqüência com que amostras de $A$. baumannii (que representam a maioria das amostras de Acinetobacter sp. estudadas) tem aumentado rapidamente nos últimos anos nos grandes hospitais brasileiros ${ }^{16}$. No presente estudo nenhuma cefalosporinas avaliada apresentou boa atividade contra esses microrganismos e a percentagem de resistência à imipenem também foi relativamente alta (14\%).

Contra bactérias gram-positivas a superioridade da cefpiroma em relação as CTGs foi mais evidente (tabela 3). A análise dos resultados obtidos com as amostras de estafilococos fica prejudicada pel o fato destas não terem si do testadas para oxacilina. A subdivisão das amostras de cada espécie em resistentes ou sensíveis à oxacilina seria importante, pois sabemos que amostras de estafilococos resistentes à oxacilina devem ser consi deradas resistentes a todos os $\beta$-lactâmicos, inclusive às cefalosporinas de amplo espectro e aos carbapenens ${ }^{15}$. De toda maneira, a cefpiroma apresentou
Tabela 3 - Atividade in vitro dos 5 -lactâmicos avaliados contra bactérias gram-positivas

\begin{tabular}{|c|c|c|c|c|}
\hline \multirow{2}{*}{$\begin{array}{l}\text { Organismo } \\
\text { (n) }\end{array}$} & \multirow[t]{2}{*}{ Antimicrobiano } & \multicolumn{2}{|c|}{$\operatorname{MICs}(\mu \mathrm{g} / \mathrm{ml})$} & \multirow{2}{*}{$\begin{array}{l}\text { Porcentagem } \\
\text { suscetívela(\%) }\end{array}$} \\
\hline & & MIC50 & MIC90 & \\
\hline Staphylococcus & Cefpiroma & 8 & 64 & 52 \\
\hline aureus & Ceftazidima & 32 & $>64$ & 34 \\
\hline \multirow[t]{3}{*}{$(141)$} & Ceftriaxona & 32 & $>64$ & 41 \\
\hline & CefotaximA & 32 & $>64$ & 45 \\
\hline & Imipenem & 1 & 64 & 61 \\
\hline \multirow{5}{*}{$\begin{array}{l}\text { Staphylococcus } \\
\text { epidermidis } \\
\text { (11) }\end{array}$} & s Cefpiroma & 1 & 16 & 73 \\
\hline & Ceftazidima & 64 & $>64$ & 36 \\
\hline & Ceftriaxona & 32 & $>64$ & 45 \\
\hline & Cefotaxima & 16 & $>64$ & 45 \\
\hline & Imipenem & 1 & 32 & 73 \\
\hline \multirow{5}{*}{$\begin{array}{l}\text { Estafilococos } \\
\text { coagulase- } \\
\text { negativos } \\
(17)\end{array}$} & Cefpiroma & 0,5 & 8 & 94 \\
\hline & Ceftazidima & 8 & $>64$ & 53 \\
\hline & Ceftriaxona & 8 & 64 & 53 \\
\hline & Cefotaxima & 8 & $>64$ & 65 \\
\hline & Imipenem & 0,12 & 32 & 88 \\
\hline \multirow{5}{*}{$\begin{array}{l}\text { Streptococcus } \\
\text { ap. }(15)^{\mathrm{b}}\end{array}$} & Cefpiroma & 0,5 & $>64$ & 80 \\
\hline & Ceftazidima & 2 & $>64$ & 73 \\
\hline & Ceftriaxona & 2 & $>64$ & 73 \\
\hline & Ceftriaxona & 4 & $>64$ & 73 \\
\hline & Imipenem & 0,5 & 8 & 87 \\
\hline \multirow{5}{*}{$\begin{array}{l}\text { Enterococcus } \\
\text { faecalis (40) }\end{array}$} & Ceftpiroma & 8 & $>64$ & 60 \\
\hline & Ceftazidima & $>64$ & $>64$ & 3 \\
\hline & Ceftriaxona & $>64$ & $>64$ & 10 \\
\hline & Cefotaxima & $>64$ & $>64$ & 10 \\
\hline & Imipenem & 2 & 4 & 90 \\
\hline \multirow{5}{*}{$\begin{array}{l}\text { Outras } \\
\text { bactérias } \\
\text { gram-positivas } \\
(10)^{c}\end{array}$} & Cefpiroma & 32 & $>64$ & 40 \\
\hline & Ceftazidima & $>64$ & $>64$ & 0 \\
\hline & Ceftriaxona & $>64$ & $>64$ & 20 \\
\hline & Cefotaxima & $>64$ & $>64$ & 20 \\
\hline & Imipenem & 2 & 8 & 80 \\
\hline \multicolumn{5}{|c|}{$\begin{array}{l}\text { a Suscetibilidade definida com MIC } \mu \mathrm{g} / \mathrm{ml}: \leq 8 \text { para as cefalosporinas e } \leq 4 \\
\text { para o Imipenem } \\
\text { b Incluindo Streptococcus pneumoniae (4 amostras), S. viridans }(3 \\
\text { amostras), estreptococos do grupo D não-enterococo ( } 2 \text { amostras), S. } \\
\text { agalactiae ( } 1 \text { amostra), S. bovis ( } 1 \text { amostra), S. pyogenes ( } 1 \text { amostra) e } \\
\text { Streptococcus sp. ( } 3 \text { amostras). } \\
\text { - Incluindo Enterococcus sp. ( } 6 \text { amostras), E. avium (1 amostra), E. hirae } \\
\text { (1 amostra) e Leuconostoc dextranicus (2 amostras). }\end{array}$} \\
\hline
\end{tabular}

atividade superior àquela apresentada pelas CTGs contra amostras de estafilococos, especialmente estafilococos coagulase negativos.

Contra $E$. faecalis também observamos uma importante superi oridade da cefpiroma em relação às CTGs (tabela 3 ), especialmente em relação à ceftazidima, que é muito utilizada na terapêutica empírica de pacientes granulocitopênicos febris. Vários estudos têm mostrado que o uso de antimicrobianos de amplo espectro com fraca atividade contra enterococos representa um fator importante para o desenvolvimento de superinfecções por esse microrganismo. Isto pode ser explicado pelo fato destes antimicrobianos eliminarem grande parte das bactérias gram-negativas da flora intestinal, propiciando a proliferação dos enterococos, principalmente quando CTGs são utilizadas em associação com aminoglicosídeos. A adição de 


\begin{tabular}{|c|c|c|c|c|}
\hline \multirow{7}{*}{$\begin{array}{l}\text { Organismo } \\
\qquad(\mathbf{n}) \\
\text { Bactérias } \\
\text { gram-positivas } \\
(570)\end{array}$} & \multirow{2}{*}{ Antimicrobiano } & \multicolumn{2}{|c|}{$\operatorname{MICs}(\mu \mathrm{g} / \mathrm{ml})$} & \multirow{2}{*}{$\begin{array}{l}\text { Porcentagem } \\
\text { suscetívela(\%) }\end{array}$} \\
\hline & & MIC50 & MIC90 & \\
\hline & Cefpiroma & 2 & 64 & 71 \\
\hline & Ceftazidima & 4 & $>64$ & 61 \\
\hline & Ceftriaxona & 16 & $>64$ & 49 \\
\hline & Cefotaxima & 16 & $>64$ & 48 \\
\hline & Imipenem & 1 & 16 & 86 \\
\hline \multirow{5}{*}{$\begin{array}{l}\text { Bactérias } \\
\text { gram-positivas } \\
(234)\end{array}$} & Cefpiroma & 8 & $>64$ & 58 \\
\hline & Ceftazidima & 64 & $>64$ & 31 \\
\hline & Ceftriaxona & 64 & $>64$ & 37 \\
\hline & Cefotaxima & 32 & $>64$ & 41 \\
\hline & Imipenem & 1 & 64 & 71 \\
\hline \multirow{5}{*}{$\begin{array}{l}\text { Total } \\
(804)\end{array}$} & Cefpiroma & 4 & 64 & 67 \\
\hline & Ceftazidima & 8 & $>64$ & 52 \\
\hline & Ceftriaxona & 16 & $>64$ & 45 \\
\hline & Cefotaxima & 16 & $>64$ & 46 \\
\hline & Imipenem & 1 & 16 & 85 \\
\hline
\end{tabular}

Suscetibilidade definida como MIC $\mu \mathrm{g} / \mathrm{ml}: \leq 8$ para as cefalosporinas e $<4$ para o Imipenem

vancomicina ao esquema terapêutico, também muito comum na terapêutica empírica do paciente granulocitopênico febril, pode levar a seleção de enterococos resistentes à vancomicina (ERV). Esse processo de sel eção de ERV foi documentado em vários hospitais americanos ${ }^{17,18}$. Já existem vários relatos na literatura, tanto de casos esporádicos quanto de surtos causados por ERV, sendo que muitas cepas são resi stentes a todos os antimi crobianos disponíveis comercialmente ${ }^{17-19}$. Além de outras medidas de controle e prevenção, muitos hospitais fizeram alterações no esquema de terapêutica empírica, substituindo a CTG por outro $\beta$ lactâmico com maior atividade contra enterococos com o intuito de dificultar a sel eção de cepas de enter ococos multirresistentes. Porém, é importante ressaltar que as cefalosporinas, mesmo as de quarta ger ação, não devem ser utilizadas no tratamento de infecções enterocócicas. A utilização da cefpiroma na terapêutica empírica do paciente granulocitopênico febril diminuiria apenas a chance de superinfecção por esse patógeno ${ }^{14,17}$. Além de ter maior ação contra enterococos a droga escolhida deve preservar a flora de anaeróbios, que desempenha papel importante nesses pacientes. Dessa maneira, a cefpiroma parece preencher os requisitos necessários para ser incluída no esquema de terapêutica empírica do paciente granulocitopênico febril. Além de apresentar excel ente ati vidade contra amostras de enterobactérias e $P$. aeruginosa, sua atividade contra estafilococos e enterococos é superior àquela apresentada pelas CTGs e sua atividade contra bactérias anaeróbias é fraca, preservando essa flora $a^{11-14}$.
Em resumo, a avaliação da atividade in vitro da cefpiroma contra amostras bacterianas isoladas em UTIs e unidades de oncohematologia de vários hospitais do Brasil, mostrou que essa nova cefalosporina possui atividade maior ou igual (dependendo da espécie) àquela apresentada pelas CTGs e muitas vezes semel hante àquela apresentada pelo carbapenem imi penem, podendo representar uma excel ente opção terapêutica para o tratamento de muitas infecções graves que ocorrem nessas unidades. Os nossos resultados também mostraram que o seu espectro de ação pode dificultar o desenvol vimento de superinfecções que tem se tornado importantes nas unidades onde foram coletadas amostras para o estudo, favorecendo assim a sua utilização em situações onde há necessidade de terapêutica empírica de amplo espectro. Acreditamos que esse antimicrobiano trará contribuição importante para o tratamento de infecções causadas por bactérias multirresistentes. Porém, é importante que o médico assistente reconheça as situações específicas onde esta droga está indicada. O intuito de estudos como este é ajudar a reconhecer as situações onde novos antimicrobianos estão indicados e as situações onde os antimicrobianos mais antigos permanecem como tratamento de primeira escolha. É importante ressaltar que o uso indiscriminado ou inadequado de novos antimi crobianos pode favorecer o aumento mais rápido da taxa de resistência, não somente ao antimi crobiano em questão como também aos antimicrobianos relacionados estruturalmente, dificultando ainda mais o combate às bactérias hospitalares multirresistentes.

\section{AGRADECIMENTOS}

Os autores agradecem Carmen Oplustil (HC-FMUSP), Fernando A. Silva (Brasília), Geraldo A. Oliveira (FCF-USP) e Waleska V. L. Farias (UNIFESP-EPM) pela valiosa contribuição na real ização dos testes laboratoriais. Este estudo recebeu auxílio financeiro da Hoechst Marion Russel do Brasil.

\section{SUMMARY}

\section{Antimicrobial activity of Cefpirome compared to other broad-spectrum Beta-Lactam drugs against 804 clinical isolates from 9 Brazilian hospitals}

OBJ ECTIVE. To eval uate the in vitro activity of the fourth-generation cephalosporin cefpirome in comparison to that of ceftazidime, ceftriaxone, cefotaxime and imipenem in a multicenter study involving nine hospitals from six cities (four States). 
Material and Method. A total of 804 isolates from patients hospitalized in either intensive care units or Oncology/ Hematology units was evaluated. The isolates were collected between J une and November of 1995, i.e. before cefpirome became commercially available in Brazil, and susceptibility tested by broth microdilution following the NCCLS procedures. All isolates resistant to cefpirome were retested by $E$-test.

RESULTS. Against Enterobacteriaceae $(n=344)$, cefpirome demonstrated an activity 2 to 32-fold higher than that of the third-generation cephalosporins (TGCS) and similar to that of imipenem. The percentages of $\mathrm{E}$ nterobacteriaceae susceptible were: $88 \%, 69 \%$ and $96 \%$ for cefpirome, TGCs and imipenem, respectively. The cefpirome spectrum was greater or equal than that of imipenem against Citrobacter freundii, Enterobacter aerogenes, Morganella morganii and Serratia marcescens. Against Acinetobacter sp. $(n=77)$, cefpirome was slightly more active than ceftazidime; however, the percentages of isolates resistant to these compounds were high $(84 \%$ and $88 \%$, respectively). The activities of cefpirome, ceftazidime and imipenem were very similar against $\mathrm{P}$. aeruginosa isolates $(n=128)$, with $M / C 50(\mathrm{mg} / \mathrm{ml}) / \mathrm{per}$ cent susceptible of $8 / 59 \%, 8 / 62 \%$ and $4 / 62 \%$ respectively. Against aerobic gram-positive bacteria, the cefpirome activity was 4 to 16 -fold higher than that of TGCs but 2 to 8-fold lower than that of imipenem.

Conclusion. The results suggest that, in Brazil, cefpirome has a spectrum of activity which is higher than that of the TGCS against aerobic gram-negative (Enterobacteriaceae and nonEnterobacteriaceae) and gram-positive bacteria and similar to that of imipenem against some Enterobacteriaceae species and $\mathrm{P}$. aeruginosa. [Rev Ass Med Brasil 1998; 44(4): 283-8.]

KEY WORDS: Cefpirome. In vitro activity. Cephal osporins. Antimicrobial susceptibility. Nosocomial bacteria. Intensive care unit.

\section{REFERÊ NCIAS BIBLIOGRÁFICAS}

1. Bush K. Characterization of ß-lactamases: groups 1, 2a, 2b, and 2b'. Antimicrob Agents Chemother 1989; 33: 264-70.

2. Richmond MH, Sykes RB. The ß-lactamases of gram-negative bacteria and their possible physiological role. Adv Microbial Physiol 1973; 9: 31-88.
3. Aronoff SC, Shlaes DM. Factors that influence the evolution of ß-lactam resistance in ß-lactamase-inducible strains of Enterobacter cloacae and Pseudomonas aeruginosa. J Infect Dis 1987, 155: 936-41.

4. Livermore DM. Mechanisms of resistance to ß-Iactam antibiotics. Scand J Infect Dis 1991; 78(Supplement): 7-16.

5. Sanders CC. ß-lactamases of gram-negative bacteria: New challenges for new drugs. Clin Infect Dis 1992; 14: 1.089-99.

6. Mariotte S, Nordmann P, Niclas MH. Extended-spectrum bIactamases in Proteus mirabilis. J Antimicrob Chemother 1995; 33: 925-35.

7. Katsanis GP, Spargo J , Ferraro MJ , Sutton L, J acoby GA. Detection of Klebsiella pneumoniae and Escherichia coli strains producing extended-espectrum ß-lactamases. J Clin Microbiol 1994; 32: 691-96.

8. Karchmer AW. Cephalosporins. In: Mandell GL, Douglas RG J r, Bennett JE eds. Principles and practice of infectious diseases. 4th ed. Churchill Livingstone Inc., 1995; p.247-64.

9. Hancock RE, Bellido F. Factors involved in the enhanced efficacy against gram-negative bacteria of fourth generation cephal osporins. J Antimicrob Chemother 1992; 29(Supp A): 1-6.

10. Nikaido H, Liu W, Rosenberg EY. Outer membrane permeability and ß-lactamasestability of dipol ar ionic cephal osporins containing methoxyimino substitutents. Antimicrob Agents Chemother 1989, 34: 337-42.

11. J ones RN, Thornsberry C, Barry AL. In vitro evaluation of HR-810, a new wide spectrum aminothiazolyl al pha-methoxyimino cephalosporin. Antimicrob Agents Chemother 1984; 25: 710-18.

12. J ones RN, Pfaller MA, Allen SD, Gerlach EH, Fuchs PC, Aldridge KE. Antimicrobial activity of cefpirome. An update compared to five third-generation cephalosporins against nearly 6,000 recent clinical isol ates from five medical centers. Diagn Microbiol Infect Dis 1991; 14: 361-64.

13. Sader HS \& J ones RN. In vitro antimicrobial activity of cefpirome against ceftazidime-resistant isolates from two multicenter studies. EurJ Clin Microbiol Infect Dis 1994; 13: 675-79.

14. Sader HS \& J ones RN. Cefalosporinas: Quatro gerações de evolução estrutural. Rev Assoc Méd Brasil 1995; 41: 144-50.

15. National Committe for Clinical Laboratory Standards (NCCLS). Approved Standard, M7-A3. Methods for dilution antimicrobial susceptibility test for bacteria that grow aerobically. 3rd ed. Villanova, PA, 1993.

16. Gales AC, Sader HS, Sinto S, Santos OP, Mendes CF. In vitro activity of ampicillin-sulbactam against multiresistant Acinetobacter baumannii strains. J Chemother 1996; 8: 416-9.

17. Edmond MB, Ober J F, Weinbaun DI, Pfaller MA, Hwang T, Sanford MD, Wenzel RP. Vancomycin-resistant Enterococcus faecium bacteremia: Risk factors for infection. Clin Infect Dis 1995; 20: 1.126-33.

18. J ones RN, Sader HS, Erwin ME and the Enterococcus Study Group. E merging multiply resistant enterococci (MRE) among clinical isolates: prevelence data from 97 medical centers. Diagn Microbiol Infect Dis 1995; 21: 85-93.

19. Sader HS, Pfaller MA, Tenover FC, Hollis RJ, J ones RN. Evaluation and characterization of multiresistant Enterococcus faecium from twelve U. S. medical centers. J Clin Microbiol 1994; 32: 2.840-42. 